\section{Case Reports in Dermatology}

Case Rep Dermatol 2016;8:42-51
This article is licensed under the Creative Commons Attribution-NonCommercial 4.0 International License (CC BY-NC) (http://www.karger.com/Services/OpenAccessLicense). Usage and distribution for commercial purposes requires written permission.

\title{
Acquired Localized Cutis Laxa due to Increased Elastin Turnover
}

\author{
Rie Harboe Nygaard ${ }^{a, b} \quad$ Scott Maynard ${ }^{b, c}$ Peter Schjerling ${ }^{a, b}$ \\ Michael Kjaer ${ }^{a, b} \quad$ Klaus Qvortrup ${ }^{d}$ Vilhelm A. Bohr ${ }^{b, c, f}$ \\ Lene J. Rasmussen $^{b, c} \quad G^{2}$ regor B.E. Jemec ${ }^{e} \quad$ Michael Heidenheim $^{e}$ \\ anstitute of Sports Medicine, Department of Orthopedic Surgery, Bispebjerg Hospital, \\ and ${ }^{\mathrm{b} C e n t e r}$ for Healthy Aging, ${ }^{\mathrm{C}}$ Department of Cellular and Molecular Medicine, \\ ${ }^{d}$ Core Facility for Integrated Microscopy, and ${ }^{\mathrm{e}}$ Department of Dermatology, Roskilde \\ Hospital, Faculty of Health and Medical Sciences, University of Copenhagen, Copenhagen, \\ Denmark; 'Laboratory of Molecular Gerontology, National Institute on Aging, \\ National Institutes of Health, Baltimore, Md., USA
}

\section{Key Words}

Irregular elastin deposition · Collagen · Dermal aging

\begin{abstract}
Cutis laxa is a rare disease characterized by abnormal skin wrinkling and laxity, due to decreased elastin synthesis or structural extracellular matrix defects. We have explored elastin metabolism in a case of adult onset cutis laxa localized to the upper body of a woman. For this purpose, we obtained skin biopsies from affected and unaffected skin areas of the patient and analyzed these with microscopy, polymerase chain reaction, western blotting and cell culture experiments. Skin from the affected area lacked elastin fibers in electron microscopy but had higher mRNA expression of elastin and total RNA. Levels of an apparent tropoelastin degradation product were higher in the affected area. Fibroblast cultures from the affected area were able to produce elastin and showed higher proliferation and survival after oxidative and UVB stress compared to fibroblasts from the unaffected area. In conclusion, we report a case of acquired localized cutis laxa with a lack of elastic fibers in the skin of the patient's upper body. The lack of elastic fibers in the affected skin was combined with increased mRNA expression and protein levels of elastin. These findings indicate that elastin synthesis was increased but did not lead to deposited elastic fibers in the tissue.
\end{abstract}

\section{KARGER}

Rie Harboe Nygaard

Institute of Sports Medicine, Bispebjerg Hospital Bispebjerg Bakke 23

DK-2400 Copenhagen NV (Denmark)

E-Mail rieharboenielsen@gmail.com 


\section{Case Reports in Dermatology}

Nygaard et al.: Acquired Localized Cutis Laxa due to Increased Elastin Turnover

\section{Introduction}

Cutis laxa is a rare disease that leads to a loss of the elastic properties of the skin and possible systemic involvement of other organ systems. The skin appears wrinkled, sagging and inelastic. The disease exists in a congenital form and in an acquired form, which can both be generalized or localized [1]. Cutis laxa is caused by defects in the synthesis of elastin or by structural defects in the extracellular matrix, and many different types of mutations have been described in the congenital form of cutis laxa [2]. The acquired form is rarer and may occur as a postinflammatory phenomenon after urticaria, for example [3, 4]. Acquired localized cutis laxa is very rare, but of particular interest as it may help elucidate the relationship between local and systemic pathogenic factors [5].

\section{Case Presentation}

We report a 30-year-old female that gradually developed severe cosmetically disabling wrinkling of the skin of her upper body over 5 years. Face, neck, breast and upper arms were the most affected areas (fig. 1), whereas the lower extremities were clinically completely unaffected. Prior to the skin changes, she had achieved an intended weight loss of $20 \mathrm{~kg}$ (BMI at the time of examination was 30). The onset of the skin changes coincided with an episode of urticaria of unexplained origin. Based on her history, clinical presentation and histopathology, the patient was diagnosed with cutis laxa. She had no family history of cutis laxa, but her mother was diagnosed as hypermobile, her grandmother (mother's side) had problems with her heart valves and her great-grandmother (mother's side) died early of heart valve insufficiency. The patient herself presented with a normal cardiography and no apparent involvement of other organ systems. We analyzed skin biopsies from the patient taken from her affected upper body (neck) and from her unaffected lower body (thigh).

\section{Materials and Methods}

\section{Skin Biopsies and Light Microscopy}

Four skin biopsies were obtained with a 4-mm dermal punch biopsy needle. The skin was sterilized and anesthetized with $2 \mathrm{ml}$ of lidocaine (1\%) before the biopsy was taken. Biopsies for cell cultures, transmission electron microscopy (TEM) and real-time RT-PCR were divided into 2 equal parts cut in the superficial-profound direction. One part was used for cell cultures, while the other was further divided for TEM and real-time RT-PCR after removal of the epidermis. Samples for TEM were fixed at $4^{\circ} \mathrm{C}$ in $2 \%$ glutaraldehyde (in 0.05 $\mathrm{M}$ phosphate buffer) and samples for real-time RT-PCR were stored at $-80^{\circ} \mathrm{C}$ until further analyses. New biopsies, following the same procedures and taken from the same areas, were obtained for light microscopy. These biopsies were kept in formalin before preparation for histological examination, underwent standard preparation and were stained using hematoxylin and eosin to demonstrate overall histology as well as Van Gieson's staining to visualize elastic fiber distribution and appearance. Unfortunately, it was not possible to stain elastin in light microscopy, and therefore these results are not shown.

Transmission Electron Microscopy

Skin samples for TEM were postfixed in $1 \% \mathrm{OsO}_{4}$ in $0.12 \mathrm{M}$ sodium cacodylate buffer (pH 7.2) for $2 \mathrm{~h}$. The specimens were then dehydrated in graded series of ethanol, trans- 
Nygaard et al.: Acquired Localized Cutis Laxa due to Increased Elastin Turnover

ferred to propylene oxide and embedded in Epon (Hexion, Houston, Tex., USA) according to standard procedures. Ultrathin sections were cut with a Reichert-Jung Ultracut E microtome, collected on Formvar membranes and stained with uranyl acetate and lead citrate. Digital images were obtained with a Philips CM100TEM (80 kV) equipped with a SIS MegaView2 camera.

\section{RNA Extraction}

Skin tissue from the patient (upper and lower body) and skin tissue from 16 young, healthy females (lower body), which served as reference, were homogenized in $1 \mathrm{ml}$ of TriReagent (Molecular Research Center, Inc., Cincinnati, Ohio, USA) containing 5 stainless steel balls of $2.3 \mathrm{~mm}$ in diameter (BioSpec Products, Inc., Bartlesville, Okla., USA), and 5 siliconcarbide sharp particles of $1 \mathrm{~mm}$ (BioSpec Products, Inc.) by shaking in a FastPrep ${ }^{\circledR}-24$ instrument (MP Biomedicals, Inc, France) at speed level 4 for $15 \mathrm{~s}$. In order to obtain complete tissue homogenization, the shaking process was repeated 3 times with ice cooling between each shaking step to avoid heating of the sample. Following homogenization, bromochloropropane was added in order to separate the samples into an aqueous and an organic phase. Following isolation of the aqueous phase, RNA was precipitated using isopropanol with glycogen for co-precipitation. The RNA pellet was then washed in ethanol and subsequently dissolved in $20 \mu \mathrm{l}$ RNAse-free water. Total RNA concentrations were determined by Ribogreen assay kit (Invitrogen).

\section{Real Time RT-PCR}

30 ng total RNA was converted into cDNA in $20 \mu \mathrm{l}$ using the SensiScript reverse transcriptase (Qiagen, Valencia, Calif., USA) and $1 \mu \mathrm{M}$ poly-dT (Invitrogen) according to the manufacture's protocol (Qiagen). For each target mRNA, $0.5 \mu \mathrm{l}$ of cDNA ( $5 \mu \mathrm{l}$ of a 10 -fold dilution) was amplified in a 25- $\mu$ l SYBR Green PCR reaction containing 1× Quantitect SYBR Green Master Mix (Qiagen) and $100 \mathrm{nM}$ of each primer (table 1). The amplification was monitored real-time using the MX3005P Real-time PCR machine (Stratagene, Santa Clara, Calif., USA). The $C_{t}$ values were related to a standard curve made with known concentrations of DNA oligos (UltramerTM oligos, Integrated DNA Technologies, Inc., Leuven, Belgium) with a DNA sequence corresponding to the sequence of the expected PCR product. Based on these $C_{t}$ values, and accounting for the PCR efficiency, the relative difference between unknown samples was determined. The specificity of the PCR products was confirmed by comparing the melt curves for the unknown samples with melt curves of the DNA oligos. GAPDH was chosen as internal control.

\section{Western Blotting}

Dermal fibroblasts from the upper affected and lower unaffected body of the patient, as well as dermal fibroblasts from the breast of a control female [age 25 years (ATCC-PCS-201012)] were grown in $100 \mathrm{~mm}^{2}$ dishes, harvested by trypsination and lysed in $0.1 \mathrm{ml}$ of $\mathrm{M}$ PER mammalian protein extraction reagent (Thermo Scientific, Rockford, Ill., USA), with added Halt protease and phosphatase inhibitor (Thermo Scientific) for $30 \mathrm{~min}$ on ice. Extracts were made from three separate plates for each of the three cells lines. These extracts were centrifuged at $12,000 \mathrm{~g}$ for $10 \mathrm{~min}$ and the supernatant was collected. The protein extracts were mixed with an equal volume of $2 \times$ tris-glycine SDS samples buffer (InvitrogenLife Technologies, Carlsbad, Calif., USA) and $20 \mu \mathrm{g}$ separated on a 12\% Mini-PROTEAN ${ }^{\circledR T G X}$ precast polyacrylamide gels (Bio-Rad, Hercules, Calif., USA). The following primary antibodies were used: tropoelastin (PR398; Elastin Products Company, Owensville, Mo., USA), collagen VI (NB120-6588; Novus Biologicals, Littleton, Colo., USA) and actin (ab1801; Abcam, 
Nygaard et al.: Acquired Localized Cutis Laxa due to Increased Elastin Turnover

Cambridge, Mass., USA) as a loading control. Secondary HRP-conjugated antibodies and Pierce ECL2 (Thermo Scientific) were used to visualize the protein bands on high performance chemiluminescence film (GE Healthcare, Pittsburgh, Pa., USA). The films were scanned and saved as 16-bit greyscale tiff files. Densitometric quantification (number of square pixels in the selected protein bands) was performed using ImageJ software (NIH, Bethesda, Md., USA).

\section{Immunofluorescence}

Dermal fibroblasts from the upper and lower body of the patient, as well as dermal fibroblasts from the breast of a control female [age 25 years (ATCC-PCS-201-012)] were seeded in covered slides (Lab-Tek II CC2 chamber slides, 4 well; Thermo Fisher, Rochester, N.Y., USA) at 50,000 cells per well and grown for 6 days. The cells were washed twice with PBS and fixed with 4\% formaldehyde (Polyscience, Warrington, Pa., USA) for $15 \mathrm{~min}$ at room temperature, and then washed twice with PBS and blocked overnight at $4{ }^{\circ} \mathrm{C}$ in $1 \%$ bovine serum albumin in PBS. The fixed cells were then incubated with rabbit anti-tropoelastin antibody (Elastin Products Company) in 1\% bovine serum albumin in PBS (diluted 1:1,000) for $2 \mathrm{~h}$ at room temperature. The cells were also incubated with normal rabbit IgG in place of the primary antibody as a background control. After two washes with PBS, the cells were incubated with Alexa Fluor 568 goat anti-rabbit antibody (Invitrogen-Life Technologies) and diluted at 1:2,000 in 1\% bovine serum albumin in PBS for $1 \mathrm{~h}$ at room temperature. The slides were mounted with DAPI $\left(4^{\prime}, 6^{\prime}\right.$-diamidino-2-phenylindole)-containing Vectashield (Vector Laboratories, Burlingame, Calif., USA) and analyzed by using a Zeiss Axiovert $200 \mathrm{M}$ microscope, with a $\times 10$ objective lens magnification for all images. Three separate experiments were performed and the immunofluorescence intensities were measured using ImageJ software.

\section{Cell Culture}

Biopsies from the upper and lower body were minced and grown in $35 \mathrm{~mm}^{2}$ dishes in DMEM GlutaMAX-1 medium (Gibco Life Technologies, Carlsbad, Calif., USA) supplemented with $10 \%$ fetal bovine serum (Gibco Life Technologies), $50 \mathrm{U} / \mathrm{ml}$ of penicillin and $50 \mu \mathrm{g} / \mathrm{ml}$ of streptomycin at $37^{\circ} \mathrm{C}$ under an atmosphere of $5 \% \mathrm{CO}_{2}$ and $20 \% \mathrm{O}_{2}$. The medium was changed every 8 days until confluence (30 days). The cells were then passaged every 8 days, seeding at $1 \times 10^{6}$ cells/plate. Growth curves and survival assays were performed on passage 4 cells. Cell proliferation assays were performed in either 96-well dishes using wst-1 cell proliferation reagent, according to the manufacturer's protocol (Roche, Mannheim, Germany), as an estimate of relative viable cell number, or in 6-well dishes using a hemocytometer to count the cells. In the case of the 96-well format, the cells were seeded (at replicates of 6 wells per cell type for each day the cell numbers were to be estimated) at either 1,000 cells per well in $100 \mu \mathrm{l}$ of medium or 5,000 cells per well in $100 \mu \mathrm{l}$ of medium, to estimate proliferation during the logarithmic phase or plateau phase, respectively. The cell numbers were estimated using wst- 1 cell proliferation reagent every day at the same time for the indicated days; $10 \mu \mathrm{l}$ of wst- 1 was added into the $100 \mu \mathrm{l}$ of medium in each well and left to incubate for $3 \mathrm{~h}$ before reading absorbance $(480 \mathrm{~nm}$ minus reading at $595 \mathrm{~nm}$ ). In the case of the 6-well format, cells were seeded at 25,000 cells per well and the cell number was counted every day at the same time (using the hemocytometer). For the cytotoxicity assays, 10,000 cells were plated in replicates of 6 onto 96-well dishes. After $24 \mathrm{~h}$ of incubation, the cells were washed in PBS and exposed to UVB at $8 \mathrm{~J} / \mathrm{m}^{2} / \mathrm{s}$ for time periods that result in the indicated doses, or to $\mathrm{H}_{2} \mathrm{O}_{2}$ (dissolved in DMEM GlutaMAX) over a range of $\mathrm{H}_{2} \mathrm{O}_{2}$ concentrations for $3 \mathrm{~h}$. At the end of the treatments, the cells were washed with PBS, and $100 \mu \mathrm{l}$ of fresh medium 
Nygaard et al.: Acquired Localized Cutis Laxa due to Increased Elastin Turnover

was added. In the case of UVB, the cells were then left to incubate for $36 \mathrm{~h}$ before adding 10 $\mu \mathrm{l}$ of wst- 1 , and in the case of the $\mathrm{H}_{2} \mathrm{O}_{2}$ assay, the wst- 1 was added right away.

\section{Results}

Transmission Electron Microscopy

Dermal collagen fibrils appeared normal from both affected and unaffected skin, but we were unable to detect any elastic fibers in the skin from the affected upper body. Normal appearing elastic fibers and collagen fibrils were found in the biopsy from the unaffected lower body (images not shown).

Skin mRNA Expression of Elastin and Collagen

The elastin mRNA expression appeared to be higher in the affected upper body (table 2), while remaining within the range of the reference samples (table 2). Collagen expression (COL1A1, COL3A1, and COL5A2) also appeared similar in the two regions (table 2). The total amount of RNA was higher in the sample from the affected upper body compared both to the unaffected lower body and the reference samples (upper body: 713, lower body: 83, reference (mean \pm standard deviation): $90 \pm 82 \mu \mathrm{g}$ total RNA per mg sample).

\section{Protein Levels of Elastin}

Results of western blotting from skin biopsies are shown in figure 2. They revealed a normal tropoelastin protein band at $\sim 75 \mathrm{kDa}$ ('tropoelastin 1') and a shortened tropoelastin protein band observed at $\sim 65 \mathrm{kDa}$ ('tropoelastin 2'). The latter was assumed to be a cleavage or degradation product from tropoelastin. Both bands were observed in cells from upper and lower body and in the control cells. However, tropoelastin 2 and the combined level of the two bands ('total tropoelastin' = tropoelastin $1+2$ ) were more abundant in cells from the upper body compared to both the lower body and the control sample. The levels of collagen VI protein (observed at $\sim 135 \mathrm{kDa}$ ) were similar in cells from the upper and lower body and from the control sample.

\section{Tissue Deposition of Elastin}

Skin fibroblasts from the affected upper body grown in culture had a greater capacity to deposit elastin in the extracellular matrix relative to the cells from the unaffected lower body (fig. 3). However, cells from both regions appeared to have irregular elastin extracellular matrix deposition relative to the control sample with normal fibroblasts (fig. 3).

\section{Cell Proliferation and Survival}

Skin fibroblasts from the affected upper body proliferated faster and reached a higher density in culture than skin cells from the unaffected lower body. Furthermore, the skin cells from the upper body had enhanced survival after UVB light exposure and oxidative stress $\left(\mathrm{H}_{2} \mathrm{O}_{2}\right)$. No apparent differences in speed or level of senescence development were observed between skin fibroblasts from the upper and lower body (data not shown).

\section{Discussion}

Our patient had clinical signs of acquired localized cutis laxa. The phenotypic presentation of the patient fitted with our microscopic findings of no elastic fibers in the skin of her 
Nygaard et al.: Acquired Localized Cutis Laxa due to Increased Elastin Turnover

affected upper body. We did, however, find higher levels of elastin mRNA expression in the affected skin area compared to the unaffected area. It could be speculated that this reflects an attempt of the fibroblasts to compensate for the low amount of elastic fibers in the tissue. This could also fit with the increased amount of total RNA in the skin cells of the affected area. From our results, we cannot ascertain why the elastin mRNA product does not end up in the tissue as elastic fibers, but since we found no elastic fibers with tissue microscopy, this discrepancy must be due to either mutation, inefficient translation of the mRNA or degradation of the protein.

In the cell cultures, we found increased protein levels of tropoelastin, but protein from the affected area seemed fragmented, and this could explain the dysfunctional nature of the elastin. Degradation of the protein has previously been described in relation to inflammatory reactions $[6,7]$, but the mechanism is described as generalized cutis laxa indicating either a local promoting factor in the diseased skin or a compensatory one in the normal skin of the patient. The cell culture experiments indicated that skin fibroblasts from the affected area behaved differently, under normal and stress conditions, from skin fibroblasts of the unaffected area. Fibroblasts taken from affected skin showed increased proliferation under typical growth conditions of $20 \%$ oxygen and enhanced cell survival after exposure to UVB light and oxidative stress $\left(\mathrm{H}_{2} \mathrm{O}_{2}\right)$. Interestingly, increases in elastin levels after ultraviolet irradiation have previously been shown to occur in normal dermal fibroblasts [8, 9]. Our findings of increased cell proliferation in the affected area without elastic fibers fit with findings in transgenic mice with a total lack of elastin (Eln-/-) and in patients with confirmed elastin haploinsufficiency, where increased cell proliferation has also been observed [10-12]. These findings suggest that elastin could have a negative influence on cell proliferation rates.

In conclusion, we report an adult female with the rare condition of localized acquired cutis laxa. The disease affected the skin of her upper body, and skin biopsies revealed an absence of elastic fibers in the dermis, despite high amounts of elastin mRNA expression and protein levels as well as high proliferative rates of skin fibroblasts.

\section{Acknowledgements}

The authors thank Anja Jokipii for her technical assistance with RNA analyses and Birgit Meinecke Nürnberg for her work with the histology stainings. This study was funded by Lundbeck Foundation, Nordea Foundation and Novo Nordic Foundation

\section{Statement of Ethics}

This work was approved by the local ethics committee, and the patient gave her written consent for the publication of this report.

\section{Disclosure Statement}

The authors report no conflicts of interest. 
Nygaard et al.: Acquired Localized Cutis Laxa due to Increased Elastin Turnover

\section{References}

$>1$

Harris RB, Heaphy MR, Perry HO: Generalized elastolysis (cutis laxa). Am J Med 1978;65:815-822. Mohamed M, Voet M, Gardeitchik T, Morava E: Cutis laxa. Adv Exp Med Biol 2014;802:161-184.

3 Chun SI, Yoon J: Acquired cutis laxa associated with chronic urticaria. J Am Acad Dermatol 1995;33:896899.

4 Lewis PG, Hood AF, Barnett NK, Holbrook KA: Postinflammatory elastolysis and cutis laxa. A case report. J Am Acad Dermatol 1990;22:40-48.

$\checkmark 5$ Riveros CJ, Gavilan MF, Franca LF, Sotto MN, Takahashi MD: Acquired localized cutis laxa confined to the face: case report and review of the literature. Int J Dermatol 2004;43:931-935.

-6 Hu Q, Reymond JL, Pinel N, Zabot MT, Urban Z: Inflammatory destruction of elastic fibers in acquired cutis laxa is associated with missense alleles in the elastin and fibulin-5 genes. J Investig Dermatol 2006;126:283-290.

7 Maruani A, Arbeille B, Machet MC, Barbet C, Laure B, Martin L, Machet L: Ultrastructural demonstration of a relationship between acquired cutis laxa and monoclonal gammopathy. Acta Derm Venereol 2010;90:406408.

$>8$ Baudry S, Lecoeuvre G, Duchateau J: Age-related changes in the behavior of the muscle-tendon unit of the gastrocnemius medialis during upright stance. J Appl Physiol 2012;112:296-304.

$\checkmark 9$ Schwartz E, Feinberg E, Lebwohl M, Mariani TJ, Boyd CD: Ultraviolet radiation increases tropoelastin accumulation by a post-transcriptional mechanism in dermal fibroblasts. J Investig Dermatol 1995;105:6569.

10 Karnik SK: A critical role for elastin signaling in vascular morphogenesis and disease. Development 2003;130:411-423.

11 Li DY, Brooke B, Davis EC, Mecham RP, Sorensen LK, Boak BB, Eichwald E, Keating MT: Elastin is an essential determinant of arterial morphogenesis Nature 1998;393:276-279.

-12 Urbán Z, Riazi S, Seidl T, Katahira J, Smoot LB, Chitayat D, Boyd CD, Hinek A: Connection between elastin haploinsufficiency and increased cell proliferation in patients with supravalvular aortic stenosis and Williams-Beuren syndrome. Am J Hum Genet 2002;71:30-44.

Rie Harboe Nygaard and Scott Maynard contributed equally to this work.

Table 1. Primer sequences

\begin{tabular}{llll}
\hline mRNA & Sense primer & Anti-sense primer & NM \\
\hline GAPDH & CCTCCTGCACCACCAACTGCTT & GAGGGGCCATCCACAGTCTTCT & NM_002046.4 \\
COL1A1 & GGCAACAGCCGCTTCACCTAC & GCGGGAGGACTTGGTGGTTTT & NM_000088.3 \\
COL3A1 & CACGGAAACACTGGTGGACAGATT & ATGCCAGCTGCACATCAAGGAC & NM_000090.3 \\
COL5A2 & CTGTGGTTCTCAAAGGGGCAAA & AGACAGTCTTGCCCACATTTCCATT & NM_000393.3 \\
Elastin & GGCTTCGGATTGTCTCCCATTTT & CCAACGTTGATGAGGTCGTGAG & NM_000501.3 \\
\hline
\end{tabular}




\section{Case Reports in Dermatology}

\begin{tabular}{l|l}
\hline Case Rep Dermatol 2016;8:42-51 \\
\hline DOI: 10.1159/000443696 & $\begin{array}{l}\text { ○ 2016 The Author(s). Published by S. Karger AG, Basel } \\
\text { www.karger.com/cde }\end{array}$ \\
\hline
\end{tabular}

Nygaard et al.: Acquired Localized Cutis Laxa due to Increased Elastin Turnover

Table 2. mRNA results

\begin{tabular}{llll}
\hline & Upper body & Lower body & Reference range \\
\hline COL1A1 & 6.34 & 8.06 & $1(0.08-12.50)$ \\
COL3A1 & 6.29 & 3.98 & $1(0.07-15.37)$ \\
COL5A2 & 2.75 & 3.60 & $1(0.11-8.97)$ \\
Elastin & 6.56 & 2.05 & $1(0.14-7.08)$ \\
\hline
\end{tabular}

All values are relative to GAPDH expression, and the geometric mean for the reference is set as 1 .
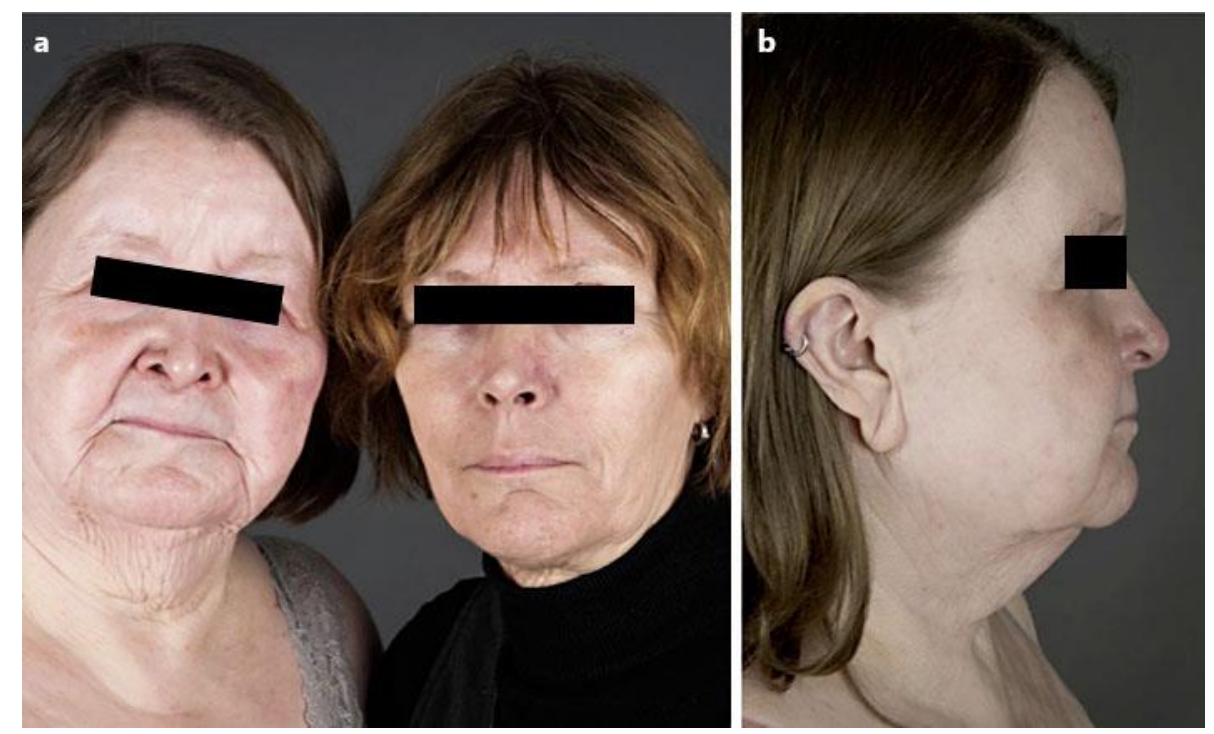

Fig. 1. Patient phenotype. a The patient (left) and her mother (right). b Note the redundant, sagging and inelastic appearance of the skin of the patient. 


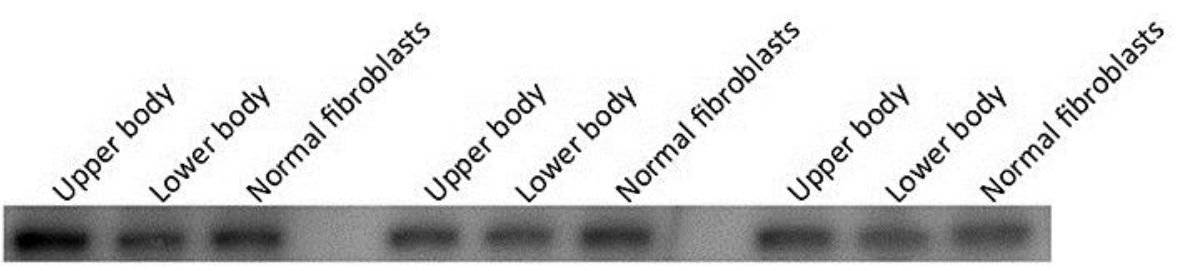

Collagen VI

Tropoelastin 1
Tropoelastin 2

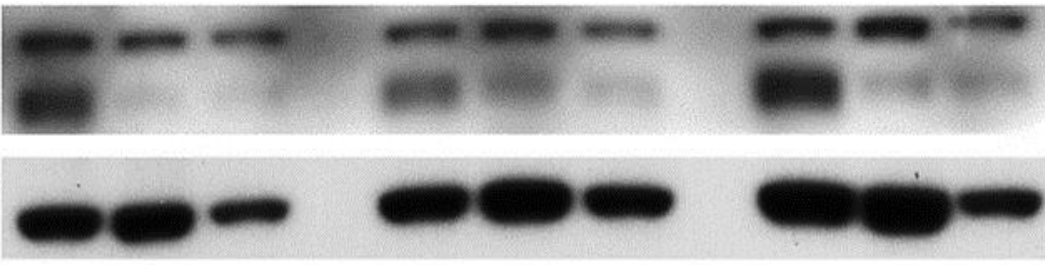

Actin

b

Tropoelastin 1

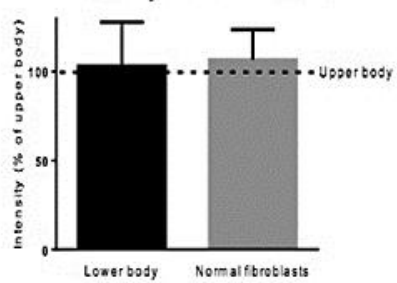

Tropoelastin 2 relative to total tropoelastin

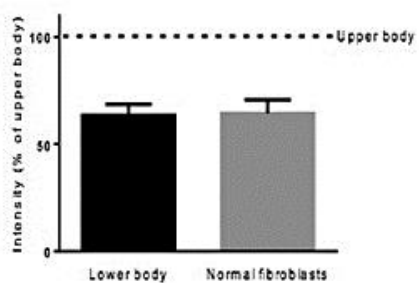

Tropoelastin 2

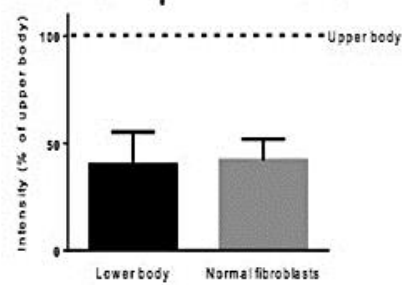

Lewerbody Normalfibroblasts

\section{Collagen VI}

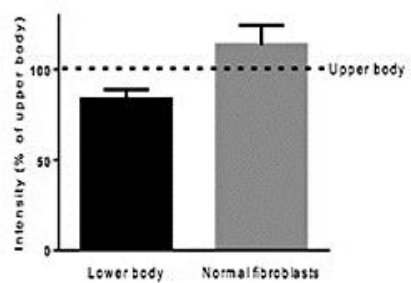

Fig. 2. a Western blotting. The figure shows western blot results from cells of the affected upper body and unaffected lower body, as well as from a control 25-year-old female (normal fibroblasts). The cells from the upper body had higher levels of the short band detected by the tropoelastin antibody (tropoelastin 2) and higher levels of the total tropoelastin (tropoelastin $1+2$ ). $\mathbf{b}$ Error bars represent the average band intensities (normalized to actin) from the three replicates shown \pm standard deviation. 


\section{Case Reports in Dermatology}

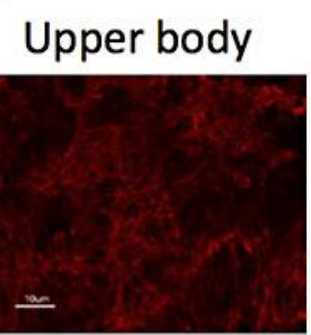

\section{Lower body}

Normal fibroblasts

b
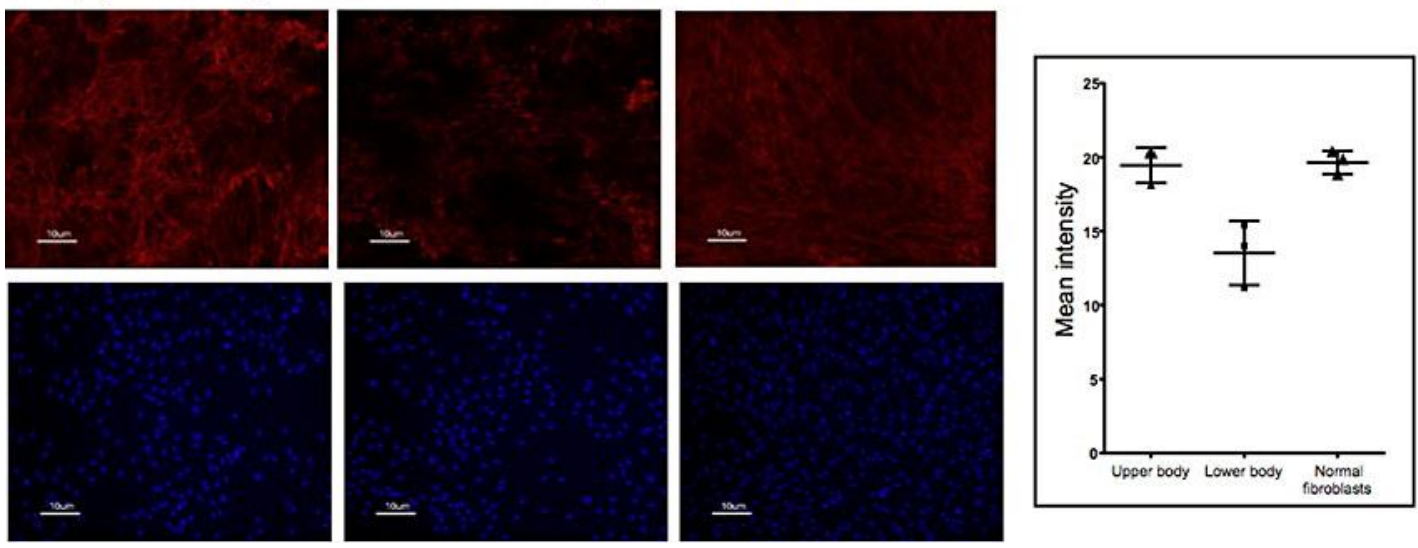

Fig. 3. a Immunohistochemistry. The skin biopsies from the affected upper and unaffected lower body, as well as from a normal female were tested for elastin deposition by immunofluorescence. Cells were grown in covered slides for 6 days and then stained for tropoelastin. Intensity was quantified by ImageJ software. b Error bars represent the average intensity (corrected for actin) of three replicates (lysates from separate plates) \pm standard deviation. The cells were also stained with DAPI to visualize cell nuclei (blue). 